\title{
Evidence for String Substructure
}

\author{
Oren Bergmant \\ Institute for Fundamental Theory \\ Department of Physics, University of Florida, Gainesville, FL, 32611, USA
}

\begin{abstract}
We argue that the behavior of string theory at high temperature and high longitudinal boosts, combined the emergence of $p$-branes as necessary ingredients in various string dualities, point to a possible reformulation of strings, as well as p-branes, as composites of bits. We review the string-bit models, and suggest generalizations to incorporate $p$-branes.
\end{abstract}

${ }^{*}$ E-mail address: oren@phys.ufl.edu 


\section{Introduction}

It has long been suspected that string theory contains far fewer degrees of freedom than implied by the world-sheet formulation, and it has been suggested that a proper formulation should make this reduction manifest [1]. A similar conclusion was reached in [2] in trying to explain the microscopic origin of black hole entropy using string theory. The area law seems to imply that the number of physical degrees of freedom in string theory has to be severely reduced at trans-Planckian energies.

Recent progress in the understanding of various dualities between string theories [3, 4] has raised the question of whether there is a more fundamental formulation that encompasses all the known string theories. Eleven dimensional M-theory [5, 6] is frequently cited as a candidate for such a formulation, even though its precise definition is not yet available. The type IIA and $E_{8} \times E_{8}$ string theories result from distinct compactifications of the eleventh dimension. The type IIB and $S O(32)$ string theories are not directly reproduced by M-theory, but their compactifications are. If, as conjectured, M-theory is a theory of membranes, it raises the question of whether string theory is really a theory of strings, or whether strings, membranes and other extended objects are equal members in a " $p$-brane democracy" [0].

Unrelated developments in string theory 8, 9, 10, 11] based on the light-cone formulation have shown that it is possible to construct composite string-bit models for strings. String-bit models are non-relativistic (Galilean invariant) matrix field theories of point particles (string-bits) in $D-2$ space dimensions and one time dimension. They allow for the formation of long closed chains, which in the continuum limit behave precisely as relativistic strings. The $D-2$ space dimensions of the string-bits become the transverse coordinates of the light-cone string, the time dimension becomes $x^{+}$, and the total mass of the chain becomes the longitudinal momentum of the string $p^{+}$. Poincaré invariance is recovered in the continuum limit at critical dimensions $(D=26$ for bosonic string, $D=10$ for superstring). These models automatically incorporate chain splitting and joining processes that mimic the closed string interactions.

At face value string-bit models are nothing more than discretizations of string theories. They regularize string theory by providing an infrared cutoff on the longitudinal momentum $p^{+}$, given by the mass of a bit. Alternatively, one can take the point of view that such a model is fundamental, and that string theory, as well as $D$-dimensional Poincaré invariance, are low energy effective features. We will argue that recent results in the study of string dualities, together with older results concerning the high temperature and high longitudinal boost behavior of string theory lend some support to this point of view.

In section 2 we review the high temperature behavior of string theory and its implication on the number of degrees of freedom in string theory. In section 3 we review the ideas of Susskind on the behavior of string theory at high longitudinal boosts, and how it is related to black hole entropy. In section 4 we review Townsend's idea of " $p$-brane democracy", that arises from string dualities, and argue that just as in "nuclear democracy" the resolution lies in compositeness. In section 5 we review string-bit models in both the bosonic and supersymmetric cases, and address the issues of the previous sections. In section 6 we suggest a generalization of the string-bit models to incorporate higher dimensional extended objects ( $p$-branes). Finally, section 7 is devoted to a discussion of the successes and limitations of our particular composite models.

\section{High Temperature}

The idea that there are degrees of freedom more fundamental than strings in string theory was first suggested in [1], by studying the high-temperature behavior of string theory. It has been 
well known from the early days of string theory that the string partition function diverges at temperatures $T>T_{H}$, where $T_{H}$ is the Hagedorn temperature given by

$$
T_{H}=\frac{1}{4 \pi \sqrt{\alpha^{\prime}}} .
$$

Rather than being a limiting temperature it is believed that $T_{H}$ is associated with a phase transition in string theory.

The appropriate target space for a finite temperature ensemble of strings is $R^{D-1} \times S^{1}$, with the radius of the $S^{1}$ given by $1 / T$. This target space supports two winding modes which become tachyonic for $T>T_{H}$. Precisely at $T=T_{H}$ these states are massless, signaling a phase transition. The classical free energy above $T_{H}$ was computed in [1], and is of the form

$$
\frac{F}{V T} \sim \frac{1}{g^{2} T}
$$

Since a genus- $k$ contribution to the free energy is of order $g^{2(k-1)}$, the above must be a genus0 contribution. But since a genus-0 Riemann surface is simply connected, i.e. has no nontrivial cycles, it cannot wind around $S^{1}$ to give a nontrivial temperature dependence. The nontrivial result (2.2) then signals a breakdown of the Riemann surface picture in the high-temperature phase. The "genus-0" Riemann surface is somehow becoming topologically nontrivial.

The large $N_{c}$ limit of QCD was invoked as support for this argument [12]. QCD is believed to undergo a deconfining phase transition at some temperature $T_{\text {dec }}$. Below $T_{\text {dec }}$ the large $N_{c}$ limit of QCD is described in terms of effective Riemann surfaces, and the free energy is computed by counting glueball and meson states. Since their spectrum is independent of $N_{c}$ for large $N_{c}$, the free energy below $T_{\text {dec }}$ is $\mathcal{O}(1)$. Above $T_{\text {dec }}$ we know that the fundamental degrees of freedom are gluons (and quarks), and therefore the free energy in the large $N_{c}$ limit is $\mathcal{O}\left(N_{c}^{2}\right)$. Since the genus$k$ contribution in the Riemann surface picture would be $\left(N_{c}^{2}\right)^{1-k}$, we see that below $T_{\text {dec }}$ there are only genus $\geq 1$ contributions, whereas above $T_{\text {dec }}$ there is a genus- 0 contribution as well. This signals a breakdown of the Riemann surface description above $T_{\mathrm{dec}}$, which is precisely what happens. Feynman diagrams involving quarks and gluons are Riemann surfaces with "holes". Confinement below $T_{\text {dec }}$ fills in the holes and gives smooth Riemann surfaces. With the presence of holes, the "genus-0 Riemann surface" is no longer simply connected, and can contribute to the free energy. In this case we know what the Riemann surface picture has to be replaced with at high temperature, namely a theory of quarks and gluons which are the composites of the low temperature degrees of freedom. We will argue that it is quite plausible that the same must be done for string theory.

The computation of the genus $\geq 1$ contributions to the free energy in string theory gives an even more suggestive result than the genus- 0 contribution. It was found that the genus- $k$ contribution is of the form

$$
\frac{F_{k}}{V T} \sim T\left(g^{2} T^{2}\right)^{k-1}
$$

It was then argued that in order to get a sensible high temperature limit, the effective coupling constant must be given by

$$
g_{\mathrm{eff}}^{2}=g^{2} T^{2},
$$

which implies that the temperature dependence of the free energy at high temperature must be

$$
\frac{F}{V T} \sim T .
$$


This is to be contrasted with the temperature dependence of the free energy of generic relativistic quantum field theories, given by

$$
\frac{F}{V T} \sim T^{D-1}
$$

indicating that there are far fewer gauge invariant degrees of freedom in string theory than in any relativistic field theory in $D>2$ dimensions, let alone in the world-sheet formulation of string theory.

The explanation given for this severe reduction in the number of degrees of freedom was that at distances shorter than $\sqrt{\alpha^{\prime}}$ string theory is no longer properly described in terms of strings or continuous world-sheets. In fact the idea that $\sqrt{\alpha^{\prime}}$ serves as a minimum distance in string theory is also suggested by T-duality, and by results on high-energy fixed-angle scattering [13]. Whatever proper formulation of string theory describes the breakdown of the Riemann surface picture at this scale, should therefore also explain the scarcity of gauge invariant degrees of freedom. This formulation clearly cannot be a relativistic field theory, unless $D=2$. A possibility not considered in [1] is that the proper formulation might be a non-relativistic (Galilean) field theory. It is well known that a gas of non-relativistic non-interacting particles exhibits a free energy of the form

$$
\frac{F}{V T} \sim T^{(D-1) / 2}
$$

so that in two space dimensions $(D=3)$ this yields the same behavior as (2.5). This is precisely the sort of system that will be considered in section 5 .

\section{High Longitudinal Boost}

Results cited in the previous section imply the breakdown of the world sheet (perturbative) formulation of string theory at a length scale $\sqrt{\alpha^{\prime}}$, and suggest that a proper formulation will contain far fewer degrees of freedom. Since these results rely on perturbative techniques, the precise length scale can be quite different. In fact an indirect argument made by Susskind [2], in trying to reconcile the behavior of strings at high longitudinal boosts with black hole entropy, suggests that that the dramatic reduction in the number of degrees of freedom is associated with a much shorter length scale given by $g \sqrt{\alpha^{\prime}}$, where $g$ is the closed string coupling constant.

The argument is based on one of the oldest known facts about string theory, namely that the physical size of a relativistic string is infinite. This is most naturally seen in the light-cone gauge formulation, where the mean square transverse separation and mean square longitudinal separation are found to diverge :

$$
\begin{aligned}
R_{\perp}^{2} \equiv\left\langle\left|x_{\perp}(\sigma)-x_{\perp}(0)\right|^{2}\right\rangle & \sim \sum_{n=1}^{\infty} \frac{1}{n} \longrightarrow \infty \\
R_{l}^{2} \equiv\left\langle\left|x^{-}(\sigma)-x^{-}(0)\right|^{2}\right\rangle & \sim \sum_{n=1}^{\infty} n \longrightarrow \infty .
\end{aligned}
$$

Realistically speaking however, any experiment designed to measure these quantities will involve a finite resolution time $\epsilon$, implying that the highest measurable frequency is $1 / \epsilon$. This provides an upper cutoff on the above sums of $n_{\max }=p^{+} / \epsilon$ in string units, where $p^{+}$is the longitudinal momentum of the string. The measured sizes will then be of the form

$$
R_{\perp}^{2} \sim \ln \frac{p^{+}}{\epsilon} \quad, \quad R_{l}^{2} \sim\left(\frac{p^{+}}{\epsilon}\right)^{2} .
$$


The above describes a peculiar growth pattern of string with longitudinal momentum, and thus with energy. As the longitudinal momentum is increased, when the string falls towards a black hole for example, the string gets longer and grows more dense, until it becomes space filling at infinite $p^{+}$(see Fig.(1)).
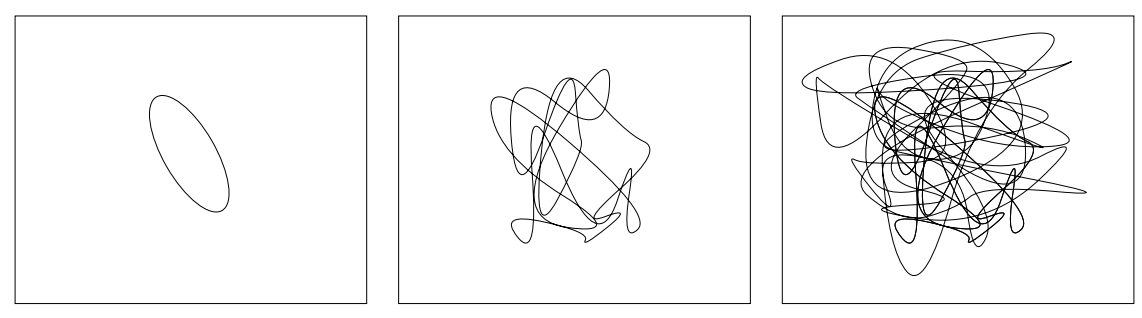

Figure 1: String growth with $p^{+}$

This conclusion assumes that the perturbative formulation used to derive (3.1) holds to arbitrarily high $p^{+}$, but it doesn't. Eventually the transverse density of string, i.e. $R_{l} / R_{\perp}^{D-2}$, will exceed $1 / g^{2}$ (in string units, i.e. $\alpha^{\prime}=1$ ), at which point interactions will contribute at $\mathcal{O}(1)$ and perturbation theory will break down. So at best we can only trust this growth pattern up to this density.

For $D=4$ the density $1 / g^{2}$ in string units is the the inverse Planck area. Susskind has suggested the possibility that the growth of string is simply cut off at the Planck scale [2]. The result is that the density grows until it reaches $\sim M_{P}^{2}$, and then remains constant as the area of the transverse region occupied by the string grows linearly with $p^{+}$, i.e.

$$
R_{\perp}^{2} \sim \frac{p^{+}}{\epsilon} .
$$

The motivation for this seemingly magical behavior of string at the Planck scale comes from a piece of non-perturbative information, namely the Beckenstein-Hawking formula for the entropy of a black hole,

$$
S_{\mathrm{BH}}=\frac{A}{4 G \hbar} .
$$

To an outside observer this entropy should arise from a counting of microstates associated with the stretched horizon of the black hole [2], which consists of infalling thermalized strings. If the string density increases to the Planck density and then remains fixed, it would imply that the black hole entropy is proportional to its area in Planck units as in (3.4).

Underlying this proposal is 't Hooft's holographic principle [14]. The Beckenstein-Hawking relation (3.4) puts an upper bound on the amount of information (entropy) that can be fit into any compact 3-dimensional region of space, whether or not there is a black hole there. Unlike our usual intuition about entropy being an extensive quantity, the entropy is bound by the area of the boundary of the region. In fact, 't Hooft has argued that the information contained in any compact region of 3-dimensional space can be encoded on its 2-dimensional boundary, at a maximum density of one bit of information per unit Planck area. The 3-dimensional world is then merely a hologram, since the true degrees of freedom come from two dimensions. Perturbative light-cone string theory offers a glimpse of such a dimensional reduction by eliminating all but the zero mode of the longitudinal direction $x^{-}$. Susskind argues that non-perturbative effects should complete this picture by explaining the maximum density of information.

Discretization of the light-cone string eliminates even the zero mode of $x^{-}$, by identifying its conjugate momentum $p^{+}$with the number of discrete bits in the string. The string is replaced 
by a discrete chain of bits, with a quantum-mechanical Hamiltonian whose continuum limit is the string's $p^{-}$. If taken seriously, the discrete chain should arise as a stable bound state of fundamental microscopic constituents in a non-relativistic field theory in $2+1$ dimensions. Thorn has devised string-bit models [8] that achieve precisely this, by utilizing 't Hoofts large $N_{c}$ limit. The microscopic bit interactions would then have to explain the origin of the maximum density, and the change in the growth pattern of the string.

\section{Duality and $p$-Brane Democracy}

The past year and a half has produced a tremendous surge of evidence for various dualities in string theory [3, 4. An important consequence of these new relations is the understanding of the role played by solitonic $p$-branes [15] in providing the non-perturbative BPS states required by duality. As BPS states these $p$-branes preserve half the supersymmetry, and must therefore carry $p$-form charges that enter the $D=10$ superalgebra as central terms. Upon compactification to four dimensions these $p$-form central charges, together with the internal momenta (Kaluza-Klein and string winding modes), provide all the possible central charges of the $D=4$ superalgebra. The latter are of a perturbative origin, whereas the former are inherently non-perturbative. However, from the point of view of the $D=4$ superalgebra there is no distinction.

The form of the superalgebra suggests that BPS-saturated $p$-branes are possible, but it is the various conjectured string dualities that make them necessary. These dualities generically map BPS states to other BPS states, and frequently map perturbative states to non-perturbative ones. The non-perturbative BPS states necessarily correspond to various wrapping modes of $p$-branes around $p$-cycles of the internal manifold.

For example in the conjectured duality of the heterotic string on $T^{4}$ and the type IIA string on $K 3$ [3, 田, the perturbative charged BPS states of the heterotic theory map into non-perturbative $\mathrm{R}$-R charged BPS states of the type IIA theory. Such states are known to exist as $p$-brane solitons in $D=10$ type IIA supergravity, with $p=0,2,4,6$, but have more recently been understood from a stringy origin as D-branes [16]. Upon compactification on $K 3$ the required states are given by the 0-brane, 2-brane wrapped around any of the 22 2-cycles of $K 3$, and 4-brane wrapped around K3.

Similarly, the conjectured U-duality of type II $D=4$ string theory [3] maps perturbative BPS states to non-perturbative ones in the same theory, and so requires the wrapping modes of the $D=10 p$-branes as well. Townsend has used this to argue that a non-perturbative formulation of string theory should exhibit a complete " $p$-brane democracy" [7], in which all $p$-branes enter as fundamental objects 1 . The string (1-brane) is singled out only because it possesses a perturbative formulation.

A possible setting in which at least the $D=10$ type IIA electric $p$-branes appear democratically is $D=11$ M-theory, whose low energy limit is $D=11$ supergravity. It has been conjectured that M-theory is in fact a supermembrane theory [17]. If so, the type IIA $p=0,1,2$ branes all arise perturbatively from that membrane. The $p=4,5,6$ branes are then their magnetic duals, and are therefore all non-perturbative. This scenario is not quite satisfactory since there is still a distinction between electric and magnetic $p$-branes, and it does not address the type IIB $p$-branes. More importantly, a successful quantization of the supermembrane is not yet available.

An amusing analogy can be drawn with the idea of "nuclear democracy", advocated in the 60's by Chew [18], and the emergence of the quark picture. Nuclear democracy is the idea that all

\footnotetext{
${ }^{1}$ Townsend has made a distinction between electrically charged $p$-branes and magnetically charged ones, the former being more fundamental than the latter.
} 
hadrons should be thought of as equally fundamental, even though particular formulations make some fundamental and others not. In the sigma model, for example, mesons are fundamental and baryons appear as solitons. The "Eightfold Way" [19] showed that the baryons and mesons fit nicely into multiplets of a symmetry group $S U(3)$. This suggested a phenomenological (but not necessarily dynamical) description of hadrons as composed of quarks transforming in a smaller representation of the symmetry group. Chew called this "nuclear aristocracy", instead of all hadrons being equally fundamental they are all equally non-fundamental. This phenomenological picture was subsequently given a dynamical setting with the formulation of QCD.

Duality is an idea similar in spirit to the eightfold way, in that it groups BPS states into "multiplets". In particular it groups fundamental string states with wrapping modes of $p$-brane solitons. In following this analogy through we suggest that that the fate of " $p$-brane democracy" is a " $p$-brane aristocracy", in which neither strings nor $p$-branes are fundamental, but composite. In the following sections we will review dynamical theories for possible constituents of strings, and suggest generalizations to $p$-branes.

\section{$5 \quad$ String-Bit Models}

The string-bit models [8, 9, 10, 11] are Galilean invariant matrix field theories in $(D-2)+1$ dimensions possessing a global $U\left(N_{c}\right)$ symmetry. The bits are thus non-relativistic particles transforming in the adjoint representation of $U\left(N_{c}\right)$. In the continuum limit one recovers $D$-dimensional lightcone string theory by interpreting the bit Hamiltonian as $p^{-}$, and the number of bits in the chain as $p^{+}$. The non-relativistic nature of the string-bit model then ensures the relativistic identity

$$
2 p^{+} p^{-}-p_{\perp}^{2}=M^{2}
$$

in the continuum string theory. Of course formation and stability of closed chains depends on the microscopic bit interactions. In fact, any attractive 2-body interaction which supports a bound state will allow chain formation. Stability, on the other hand, is only guaranteed for certain supersymmetric string-bit models [10], but universality is expected [11]. Splitting and joining interactions of strings are manifest in these models as $\mathcal{O}\left(1 / N_{c}\right)$ corrections, leading to the identification of the string coupling constant as

$$
g \sim 1 / N_{c}
$$

\subsection{Bosonic Model}

Let us begin by reviewing the bosonic string-bit model of [ [8]. We second quantize the string-bits by introducing a field $\phi(x)_{\alpha}^{\beta}$ and its hermitian conjugate $\phi^{\dagger}(x)_{\alpha}^{\beta}$, satisfying the canonical commutation relation

$$
\left[\phi(x)_{\alpha}^{\beta}, \phi^{\dagger}(y)_{\gamma}^{\delta}\right]=\delta_{\alpha}^{\delta} \delta_{\gamma}^{\beta} \delta(x-y)
$$

The singlet operators

$$
A^{\dagger}\left(x_{1}, \ldots, x_{N}\right)=N_{c}^{-N / 2} \operatorname{Tr}\left[\phi^{\dagger}\left(x_{1}\right) \cdots \phi^{\dagger}\left(x_{N}\right)\right]
$$

behave as creation operators for $N$-bit chains in the large $N_{c}$ limit? Consider the following stringbit Hamiltonian

$$
H=\frac{1}{2 m} \int d x \operatorname{Tr}|\nabla \phi|^{2}+\frac{T_{0}^{2}}{2 m N_{c}} \int d x d y V(x-y) \operatorname{Tr}\left[\phi^{\dagger}(x) \phi^{\dagger}(y) \phi(y) \phi(x)\right] .
$$

\footnotetext{
${ }^{2} A^{\dagger}$ and $A$ satisfy the canonical commutation relation only in the limit $N_{c} \rightarrow \infty$.
} 
Acting on the closed chain state

$$
|\psi\rangle=\int d x_{1} \cdots d x_{N} A^{\dagger}\left(x_{1}, . ., x_{N}\right)|0\rangle \psi_{N}\left(x_{1}, . ., x_{N}\right)
$$

it gives

$$
\begin{aligned}
H|\psi\rangle & =\int d x_{1} \cdots d x_{N}\left\{A^{\dagger}\left(x_{1}, . ., x_{N}\right)|0\rangle \sum_{k=1}^{N}\left[-\nabla_{k}^{2} / 2 m+\frac{T_{0}^{2}}{2 m} V\left(x_{k+1}-x_{k}\right)\right] \psi_{N}\left(x_{1}, . ., x_{N}\right)\right. \\
& \left.+\frac{T_{0}^{2}}{2 m N_{c}} \sum_{k=1}^{N} \sum_{l \neq k, k+1} A^{\dagger}\left(x_{k+1}, . ., x_{l-1}\right) A^{\dagger}\left(x_{l}, . ., x_{k}\right)|0\rangle V\left(x_{k}-x_{l}\right) \psi_{N}\left(x_{1}, . ., x_{N}\right)\right\} .
\end{aligned}
$$

In the limit $N_{c} \rightarrow \infty$ the second term drops out, and one can associate a first-quantized Hamiltonian to an $N$-bit chain :

$$
H_{N}=\frac{1}{2 m} \sum_{k=1}^{N}\left[p_{k}^{2}+T_{0}^{2} V\left(x_{k+1}-x_{k}\right)\right] .
$$

The above describes the dynamics of a bare closed chain, provided of course that the nearestneighbor potential is strong enough to bind. For the simple case in which the potential is harmonic,

$$
V(x)=x^{2}
$$

this system is exactly soluble by Fourier transforming. The excitation energies of the collective modes of the chain are given by

$$
E_{n}=\frac{2 T_{0}}{m} \sin \frac{n \pi}{N}
$$

where $n$ is the mode number. In the continuum limit given by

$$
m \rightarrow 0, N \rightarrow \infty, m N=\text { fixed }
$$

the Hamiltonian becomes

$$
H_{N} \rightarrow \frac{1}{2 T_{0}} \int_{0}^{m N / T_{0}} d \sigma\left[\mathcal{P}(\sigma)^{2}+T_{0}^{2} x^{\prime}(\sigma)^{2}\right],
$$

and the excitation energies for the finite $n$ modes become

$$
E_{n} \rightarrow \frac{2 \pi n T_{0}}{m N}
$$

The finite $(N-n)$ modes have energies given by (5.13) with $n$ replaced by $(N-n)$. All other modes have an infinite excitation energy, and therefore decouple in the continuum limit. The Hamiltonian (5.12) corresponds precisely to the light-cone Hamiltonian $\left(p^{-}\right)$of the bosonic string, if one identifies the longitudinal momentum of the string as

$$
p^{+}=m N \text {. }
$$

In the continuum limit this is a continuous variable, so it defines a new coordinate $x^{-}$as its canonical conjugate. From the point of view of string theory this is the longitudinal coordinate of the string. The string-bit model with $N_{c} \rightarrow \infty$ and a harmonic potential is thus a straightforward discretization of free light-cone bosonic string theory. 
The appearance of an $\mathcal{O}\left(1 / N_{c}\right)$ term in (5.7), corresponding to the splitting of a single chain into two chains, suggests an alternative point of view in which string-bits are fundamental, and interacting (light-cone) string theory arises as a low energy effective theory. From this point of view the mass of string-bits $(m)$ is non-zero, and one studies finite size $(N)$ chains. At characteristic energies $\ll T_{0} / m$ only modes with $n \ll N$ are probed, so the effective spectrum is stringy, as in (5.13). In this limit the $\mathcal{O}\left(1 / N_{c}\right)$ chain interactions become string interactions, so the string coupling constant is identified with $1 / N_{c}$.

This simple example shows that it is possible in principle to construct a composite picture of strings in the light-cone formulation. There are however four possible drawbacks to this particular model :

1. It is unstable against the decay of chains into smaller chains.

2. It has strong long range interactions between chains.

3. The microscopic string-bit interaction leading to chain formation is not unique.

4. It depends strongly on the light-cone formulation of string theory.

The first point can be understood from the continuum point of view as the tachyonic instability. In the discrete model it is simply a consequence of energetics. The ground state energy of a long closed chain is generically given by

$$
E_{\mathrm{G} . \mathrm{S} .}=(D-2) \sum_{n=1}^{N-1} E_{n}=\frac{D-2}{m}\left[a N+\frac{b}{N}+O\left(\frac{1}{N^{2}}\right)\right] .
$$

The first term is clearly the same for a single chain of $N$ bits and two chains of $N_{1}$ and $N_{2}$ bits, with $N_{1}+N_{2}=N$. For long chains, the nature of the true ground state then depends on the second term. In the above model $b<0$, so $E_{0, N}>E_{0, N_{1}}+E_{0, N_{2}}$, implying that the chain is unstable to decay into two smaller chains. In the continuum limit $b$ becomes the mass squared of the tachyon.

The second point follows from the fact that the chain splitting term in (5.7) is weighted by the microscopic potential $V(x-y)$, which in the harmonic case diverges at infinite separation. This makes a definition of the S-matrix problematic. For a well defined S-matrix we need a short range interaction, which is however still strong enough to bind. In addition the low energy (long wavelength) properties of the chains should still approximate continuous strings. Namely, the low energy collective excitations should reproduce the string modes in the continuum limit. The idea of universality suggests that the long-wavelength properties of the discrete system are to some extent independent of the short-distance physics. We therefore expect a large universality class of microscopic interactions, that includes the harmonic potential, to yield an approximate description of free strings at low energy. In particular the interaction can be short range. Thorn has explored the possibility of a zero-range interaction, and has computed numerically the string tension arising from such a model [9]. Universality is an appealing idea, but it remains unproven in bosonic string-bit models.

The third point is made clear by adding the following interaction term to the Hamiltonian (5.5),

$$
\frac{\lambda}{N_{c}} \int d x d y U(x-y): \operatorname{Tr}\left[\phi^{\dagger}(x) \phi(x) \phi^{\dagger}(y) \phi(y)\right]:,
$$

where $\lambda$ and $U(x)$ are arbitrary. Unlike the original interaction, the annihilation operators do not appear consecutively in the matrix product. Consequently, the action of this operator on the closed 
chain state will only produce $\mathcal{O}\left(1 / N_{c}\right)$ terms, which will not contribute in the $N_{c} \rightarrow \infty$ limit to the first-quantized Hamiltonian (5.8). As far as free closed chains are concerned then, the microscopic string-bit interaction is determined only up to such a term. This term will contribute to chain interactions, so one might hope to fix the ambiguity by comparing chain scattering amplitudes in the continuum limit to known results in string theory.

The fourth point may be significant and may be irrelevant, depending on your point of view. Since the world-sheet (Riemann surface) picture breaks down at high temperature, world-sheet coordinate invariance is at best a low energy principle. If it is, then any reformulation of string theory appropriate for describing the high energy behavior should reduce to the gauge invariant world-sheet formulation at low energy. Constituents are not a good candidate since a constituent picture does not arise naturally in gauges other than light-cone. However, in a formulation that is free of unphysical states from the outset there is no need for gauge (world-sheet coordinate) invariance. Of course, such a formulation would generally be much more complicated, and not Poincaré invariant. In the string-bit models both world-sheet coordinate invariance and Poincaré invariance are abandoned in favor of $(D-2)+1$-dimensional Galilean invariance and a global $U\left(N_{c}\right)$ symmetry, such that the physical (a.k.a. gauge invariant) properties of strings are reproduced at low energy. In addition, Poincaré invariance is recovered as an effective low energy symmetry at the critical dimension. The fact that the low energy dynamics of chains seem to arise from a light-cone gauge fixed world-sheet theory is purely accidental from this point of view, and is more a property of two dimensional field theory than of strings. In fact we will suggest a composite formulation of $p$-branes in the next section, whose low energy limit gives the the physical (transverse) modes, but cannot be consistently derived from a covariant formulation, as the latter does not possess sufficient gauge symmetry.

\subsection{Supersymmetric Models}

It is well known that the stability issue gets resolved in string theory by making it supersymmetric. The ground state of the superstring (IIA, IIB, or Heterotic) is massless. We follow a similar path for string-bits by associating an additional degree of freedom, "statistics", to the bits, distinguishing bosonic bits from fermionic bits. This will give rise to "statistics" waves traveling on the long chains, in addition to the usual coordinate "phonon" waves. Supersymmetry is implemented by extending the Galilean symmetry of the bosonic string-bit model by gradation to an $\mathcal{N}=1$ SuperGalilean symmetry [10, 20]. The supercharges $\mathcal{Q}^{A}, \mathcal{R}^{A}$ transform as real $D-2$-component spinors under the $S O(D-2)$ subgroup of the Galilei algebra, and satisfy the following anti-commutation relations

$$
\begin{gathered}
\left\{\mathcal{Q}^{A}, \mathcal{Q}^{B}\right\}=m N \delta^{A B} \quad, \quad\left\{\mathcal{Q}^{A}, \mathcal{R}^{\dot{B}}\right\}=\frac{1}{2} \boldsymbol{\alpha}^{A \dot{B}} \cdot \mathbf{P}, \\
\left\{\mathcal{R}^{\dot{A}}, \mathcal{R}^{\dot{B}}\right\}=\delta^{\dot{A} \dot{B}} H / 2
\end{gathered}
$$

where $\alpha^{i}$ are the transverse $D$-dimensional Dirac $\alpha$-matrices corresponding to the embedding $S O(D-2) \times S O(1,1) \subset S O(D-1,1)$. Undotted indices correspond to positive $S O(1,1)$ chirality and dotted indices correspond to negative $S O(1,1)$ chirality. Consequently $\mathcal{Q}$ and $\mathcal{R}$ will combine into a single supercharge transforming as a spinor of $S O(D-1,1)$ upon recovery of the longitudinal dimension $x^{-}$in the continuum limit.

Models were constructed in $1+1$ [11], $2+1$ and $8+1$ [10] dimensions, underlying $D=3,4$ and 10 type IIB superstring. In the lower dimensional cases extra degrees of freedom must be eventually 
added to make the string critical $]^{3}$. In the $2+1$ and $8+1$ models [10] the last relation in (5.17) could not be implemented. There were additional terms not proportional to $\delta^{\dot{A} \dot{B}}$. In the $1+1$ dimensional model there are no indices, so the full superalgebra closes by default. In this model the supercharges are given by

$$
\begin{aligned}
\mathcal{Q}= & \sqrt{\frac{m}{2}} \int d x \operatorname{Tr}\left[e^{i \pi / 4} \phi^{\dagger}(x) \psi(x)+\text { h.c. }\right] \\
\mathcal{R}= & -\frac{1}{2 \sqrt{2 m}} \int d x \operatorname{Tr}\left[e^{-i \pi / 4} \phi^{\dagger}(x) \psi^{\prime}(x)+\text { h.c. }\right] \\
& +\frac{1}{2 N_{c} \sqrt{2 m}} \int d x d y W(y-x) \\
& \times \operatorname{Tr}\left[e^{-i \pi / 4} \phi^{\dagger}(x) \rho(y) \psi(x)+\text { h.c. }\right],
\end{aligned}
$$

where $\psi_{\alpha}^{\beta}$ is the fermionic annihilation operator, $\rho_{\alpha}^{\beta}=\left[\phi^{\dagger} \phi+\psi^{\dagger} \psi\right]_{\alpha}^{\beta}$, and $W(x-y)$ is a quantummechanical superpotential. The $1+1$ dimensional superalgebra is given by:

$$
\{\mathcal{Q}, \mathcal{Q}\}=m N,\{\mathcal{Q}, \mathcal{R}\}=-P / 2,\{\mathcal{R}, \mathcal{R}\}=H / 2 \text {. }
$$

The last equation can be taken as the definition of the Hamiltonian, which is given by

$$
\begin{aligned}
H= & \frac{1}{2 m} \int d x \operatorname{Tr}\left[|\nabla \phi|^{2}+|\nabla \psi|^{2}\right] \\
+\frac{1}{2 m N_{c}} \int d x d y & \left\{\left[W^{2}(y-x)+W^{\prime}(y-x)\right] \operatorname{Tr} \phi^{\dagger}(x) \rho(y) \phi(x)\right. \\
& +\left[W^{2}(y-x)-W^{\prime}(y-x)\right] \operatorname{Tr} \psi^{\dagger}(x) \rho(y) \psi(x) \\
& \left.\quad+W^{\prime}(y-x) \operatorname{Tr}\left[i \phi^{\dagger}(x) \phi^{\dagger}(y) \psi(y) \psi(x)+\phi^{\dagger}(x) \psi^{\dagger}(y) \phi(y) \phi(x)+\text { h.c. }\right]\right\} \\
+ & \frac{1}{2 m N_{c}^{2}} \int d x d y d z W(y-x) W(z-x): \operatorname{Tr}\left[\phi^{\dagger}(x) \rho(z) \rho(y) \phi(x)+\psi^{\dagger}(x) \rho(z) \rho(y) \psi(x)\right]: \\
+ & \frac{1}{4 m N_{c}^{2}} \int d x d y d z W(y-x) W(y-z) \times \\
& \quad \times: \operatorname{Tr}\left[\phi^{\dagger}(x)\left[\phi(z) \psi^{\dagger}(z), \rho(y)\right] \psi(x)-i \phi^{\dagger}(x)\left[\psi(z) \phi^{\dagger}(z), \rho(y)\right] \psi(x)+\text { h.c. }\right]:, \quad(5.20)
\end{aligned}
$$

where the commutators in the last integral refer only to matrix ordering. The last two integrals represent 3-body interactions. These were absent in the bosonic model (5.5), but are required here by supersymmetry. This model is invariant under the entire supersymmetry since

$$
[H, \mathcal{Q}]=[H, \mathcal{R}]=0
$$

Acting with this Hamiltonian on the supersymmetric chain state

$$
\begin{aligned}
|\Psi\rangle & =\int d x_{1} d \theta_{1} \cdots d x_{N} d \theta_{N} \Psi\left(x_{1}, \theta_{1} \ldots, x_{N}, \theta_{N}\right) \\
& \times \operatorname{Tr}\left(\left[\phi^{\dagger}\left(x_{1}\right)+\psi^{\dagger}\left(x_{1}\right) \theta_{1}\right] \cdots\left[\phi^{\dagger}\left(x_{N}\right)+\psi^{\dagger}\left(x_{N}\right) \theta_{N}\right]\right)|0\rangle
\end{aligned}
$$

\footnotetext{
${ }^{3}$ An implementation of this idea was first suggested in [21].
} 
and taking the limit $N_{c} \rightarrow \infty$ yields the following first quantized Hamiltonian :

$$
\begin{aligned}
H_{N} & =\frac{1}{2 m} \sum_{k=1}^{N}\left\{p_{k}^{2}+W^{2}\left(x_{k+1}-x_{k}\right)\right. \\
& +W^{\prime}\left(x_{k+1}-x_{k}\right)\left[\theta_{k} \pi_{k}-\pi_{k} \theta_{k}\right. \\
& \left.\left.+\pi_{k+1} \theta_{k}-\theta_{k+1} \pi_{k}-i\left(\theta_{k} \theta_{k+1}+\pi_{k} \pi_{k+1}\right)\right]\right\}
\end{aligned}
$$

where $\pi_{k} \equiv \partial / \partial \theta_{k}$. Note that $W(x)$ is not the usual superpotential of supersymmetric quantum mechanics (SUSY QM) [22], for which the potential would be given by

$$
V(x)=W^{2}(x) \pm W^{\prime}(x) .
$$

That superpotential was restricted to having an odd number of nodes by virtue of the zero ground state energy. As can be seen in our Hamiltonian (5.23), the identification (5.24) does not hold in general, and it is not yet clear what, if any, restrictions are placed on $W(x)$. For the special case in which $W(x)$ is an odd function however, the two-bit $(N=2)$ sector is equivalent to the SUSY QM of [22], and (5.24) holds. A comprehensive study of the two-bit sector will be carried out elsewhere. For now we restrict ourselves to the linear superpotential

$$
W(x)=T_{0} x
$$

which defines the supersymmetric extension of the harmonic string-bit model. In this extension the excitation energies of the collective statistics modes are the same as the phonon modes,

$$
E_{n}=\frac{2 T_{0}}{m} \sin \frac{n \pi}{N}
$$

but they contribute to the ground state energy with opposite sign, giving an exact cancelation,

$$
E_{\text {G.S. }}=0 \text {, }
$$

for any length chain. The exact vanishing of the ground state energy guarantees stability at the discrete level, which is a much stronger result than the absence of the tachyon in continuum superstring theories. The latter requires only that $b=0$ in (5.15), whereas the former states that all the terms in (5.15) vanish. This is crucial if the bit picture is to provide a fundamental formulation of superstring theory.

Upon changing the fermionic variables to

$$
\begin{aligned}
& S_{k}=\frac{1}{\sqrt{2}}\left(\theta_{k}+\pi_{k}\right) \\
& \tilde{S}_{k}=\frac{i}{\sqrt{2}}\left(\theta_{k}-\pi_{k}\right),
\end{aligned}
$$

the continuum limit gives

$$
H_{N} \rightarrow \frac{1}{2 T_{0}} \int_{0}^{p^{+} / T_{0}} d \sigma\left[\mathcal{P}(\sigma)^{2}+T_{0}^{2} x^{\prime}(\sigma)^{2}-i T_{0} S(\sigma) S^{\prime}(\sigma)+i T_{0} \tilde{S}(\sigma) \tilde{S}^{\prime}(\sigma)\right]
$$

This is precisely the light-cone Hamiltonian of the three dimensional free type IIB superstring, if one interprets $S$ and $\tilde{S}$ as the right and left moving fermions respectively. We therefore refer to this supersymmetric string-bit model as a type IIB superstring-bit model. Note that the supersymmetry 
has doubled to the required $\mathcal{N}=2$ in the continuum limit. The original supercharges of the superstring-bit model $\mathcal{Q}, \mathcal{R}$ correspond to a sum of the right and left moving supercharges of the continuum type IIB superstring theory. One still has to check the finite $N_{c}$ interaction terms, to see if they agree with the known continuum results, or if additional terms need to be added to this model.

The recovery of superstringy physics at low energy was manifest for the supersymmetric harmonic model, but is actually a result of the fact that the ground state energy vanished and the excitation gap was finite. Universality suggests that low energy superstringy physics is expected to hold more generally for any supersymmetric model satisfying these two properties. On the other hand the supersymmetric harmonic model still gives rise to long range interactions between chains, precluding a well defined S-matrix. One would therefore like to find short-range interactions leading to well defined S-matrices, within the universality class of the harmonic model.

For 1+1 dimensional superstring-bit models [11], we were able to prove a restricted form of universality in perturbation theory, and argued that the "smallness" of the zero-energy representation of the superalgebra implies that it can be extended beyond perturbation theory. It was shown that deforming the superpotential away from linearity

$$
W(x)=T_{0} x+\delta W(x),
$$

where

$$
|\delta W(x)| \ll T_{0}|x| \text { for }|x|<\sqrt{\alpha^{\prime}},
$$

does not change the results (5.26), (5.27) qualitatively, but only renormalizes the string tension,

$$
T_{0} \rightarrow T_{0}+\left\langle\delta W^{\prime}\left(x_{2}-x_{1}\right)\right\rangle,
$$

where the expectation value is computed in the zeroth order (super-harmonic) chain state. In fact

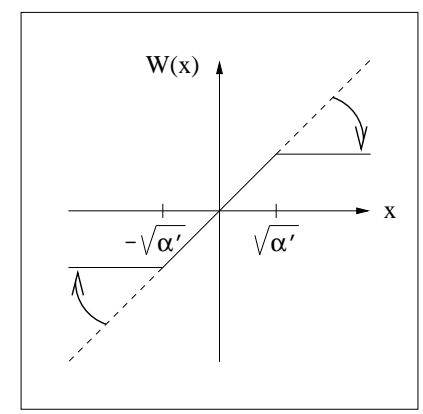

Figure 2: Superpotential deformation

with the deformation in Fig.(2) we achieve the required short-range interaction, while still keeping the stringy long-distance physics. Actually this deformation is not quite enough to guarantee the clustering property required for a well defined S-matrix. That is achieved by further replacing $\rho(y)$ with $\rho(y)-\sigma(y)$ in eq. (5.18), where $\sigma_{\alpha}^{\beta}=:\left[\phi \phi^{\dagger}-\psi \psi^{\dagger}\right]_{\alpha}^{\beta}$ :. A successful implementation of these ideas for the higher dimensional superstring-bit models, in particular ones leading to critical superstrings, is under current study.

The superstring-bit model we presented above is most likely incomplete, even in the simple $D=3$ case. In particular it seems to lack the richness of structure of the superstring interactions as given in [23]. A detailed analysis of the perturbative chain interactions in this model will be carried out elsewhere. Instead, we will try to understand the generic features of such string-bit models under the extreme conditions addressed in sections 2,3. 


\subsection{Extreme Conditions}

At high temperature the Riemann surface description of string theory breaks down, and a phase transition is expected to occur. It was shown that in such a transition "holes" must appear in the world-sheet, to support a genus-0 free energy. String-bit models offer an explicit realization of this transition. It corresponds to a dissociation transition, in which the chain breaks into bits (Fig.(3)). The critical temperature should be related to the energy scale at which the bonds between the bits
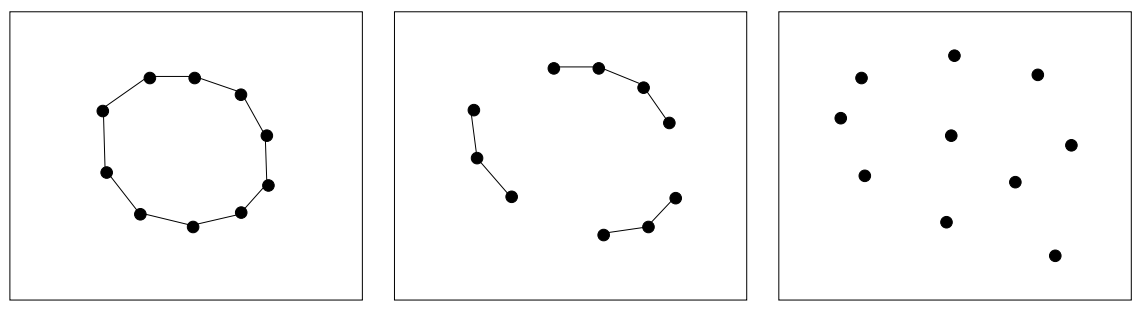

Figure 3: Dissociation transition

break, in other words the binding energy of two bits in a chain :

$$
T_{c} \sim E_{B} \sim T_{0} / m
$$

Not surprisingly, this temperature is quite different than the Hagedorn temperature $T_{H} \sim \sqrt{T_{0}}$. Recall that the Hagedorn transition was derived using perturbative techniques. One can therefore trust the qualitative result of the phase transition, but not necessarily the precise value of the critical temperature.

The high temperature phase is a gas of non-relativistic weakly interacting particles, with a free energy given by eq. (2.7) :

$$
\frac{F}{V T} \sim T^{(D-1) / 2}
$$

For a $2+1$ dimensional string-bit model $(D=4)$, appropriate for describing the microscopic building blocks of a 4-dimensional string theory, this agrees with Atick and Witten's result (2.5).

The other extreme condition is a large longitudinal boost, leading to a high density of string (Fig.(1)). From the string-bit point of view this corresponds to adding bits successively to a chain (Fig.(4)). The chain gets longer because the length of a bond between nearest neighbor bits is fixed by the microscopic bit interaction. For the harmonic model the bond length is given by $\sqrt{\alpha^{\prime}}$, so the length of an $N$-bit chain is $N \sqrt{\alpha^{\prime}}$. The amount of space occupied by the chain can be estimated by the mean square distance between two well separated bits in the chain,

$$
\left\langle\Delta x^{2}\right\rangle=\left\langle\left(x_{i}-x_{j}\right)^{2}\right\rangle,
$$

where $i-j \sim \mathcal{O}(N)$ for large $N$. In the limit $N_{c} \rightarrow \infty$, appropriate for reproducing the behavior of free string, we can ignore the non-nearest neighbor interactions, and use the ground state of the bare chain Hamiltonian $H_{N}$ for this purpose. The result is given by

$$
\left\langle\Delta x^{2}\right\rangle \sim \alpha^{\prime} \ln N
$$

in agreement with the free string result. The density of string-bits then grows like

$$
\rho \sim \frac{N}{\left(\alpha^{\prime} \ln N\right)^{(D-2) / 2}} .
$$



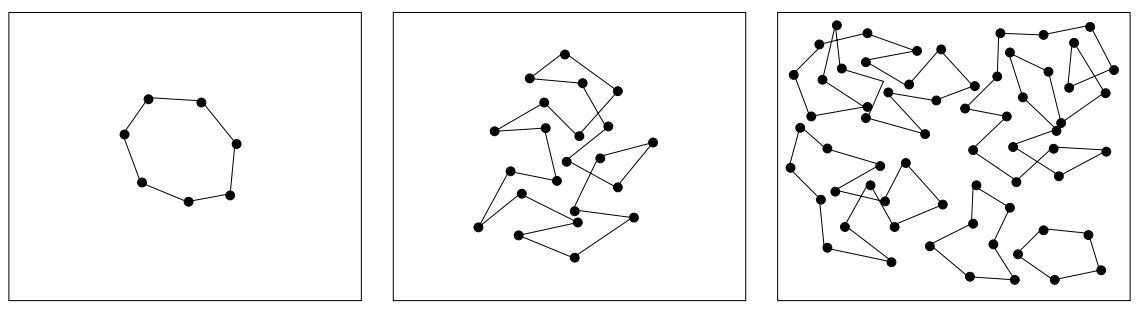

Figure 4: Adding bits to a chain

As the density of string-bits grows, many non-nearest neighbor bits will come close, and nonnearest neighbor interactions will become important. Eventually the bare chain approximation used to derive (5.37) will fail, just as the free string approximation did in section 3. Chains will join and split freely, resulting in an ensemble of chains of varying lengths. The correction to eq. (5.37), and with it the high density behavior of the chain, will depend crucially on the nature of the non-nearest neighbor interactions. In particular, an attractive non-nearest neighbor interaction will tend to make the chain more dense, whereas a repulsive one will tend to spread it out. A hard core repulsion ("elbows") between non-nearest neighbors would imply a maximum density of bits, $\rho_{\max }$, depending on the size of the core.

In the bosonic model given by eq. (5.5) with $V(x)$ an attractive potential, the non-nearest neighbor interaction is $\sim V(x) / N_{c}^{2}$, and therefore also attractive. By adding to this model the term in eq. (5.16) with the same potential and $\lambda=-T_{0} / 2 m$, one can make the non-nearest neighbor interaction repulsive $\sim-V(x) / N_{c}^{2}$, while still maintaining an attractive nearest-neighbor interaction. Since $V(x)$ must be short range, the non-nearest neighbor repulsion will have a finite length scale associated with it, even though it is not necessarily hard core. Indeed, the superpotential considered at the end of section 5.2 will yield a non-nearest neighbor interaction range of $\sim \sqrt{\alpha^{\prime}}$, and a strength of $\sim T_{0} /\left(m N_{c}^{2}\right)$, coming from virtual chain corrections to the bare chain. It is yet to be determined if this soft-core repulsion gives $\rho_{\max } \sim N_{c}^{2}\left(\alpha^{\prime}\right)^{(2-D) / 2}$, as suggested by Susskind.

Another possible origin of a maximum density of string-bits is a hard core repulsion in the potential $V(x)$ itself. An example of a superpotential which would achieve this is sketched in Fig.(5). We do not yet know however if the universality arguments made in 11] can be extended to this superpotential.

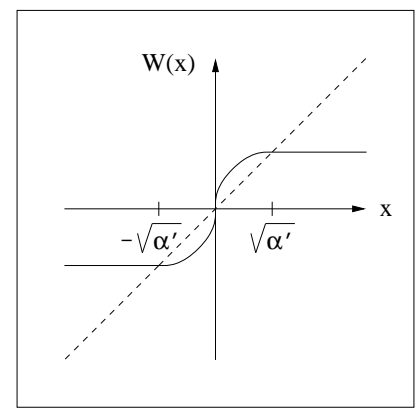

Figure 5: Hard core superpotential 


\section{6 p-Branes From Bits?}

Finally we would like to suggest a generalization of the string-bit models to incorporate other extended objects, whose low energy limits are $p$-branes. For concreteness we concentrate on bosonic membranes. We leave the extension to higher $p$-branes and supersymmetry for future work.

String-bits could be thought of as particles with a pair of "legs" (Fig.(6a)), that could be attached to other bits consecutively to form a chain. The generalization to membrane-bits then consists of adding more "legs" pairwise. Closed membranes will be replaced with compact twodimensional lattices of bits. The types of lattices one can form will depend on how many "legs" the bits have, but the continuum limit should be independent of this. Let us assume membrane-bits to have two pairs of legs (Fig.6b)). This means that membrane-bit fields have two pairs of indices,
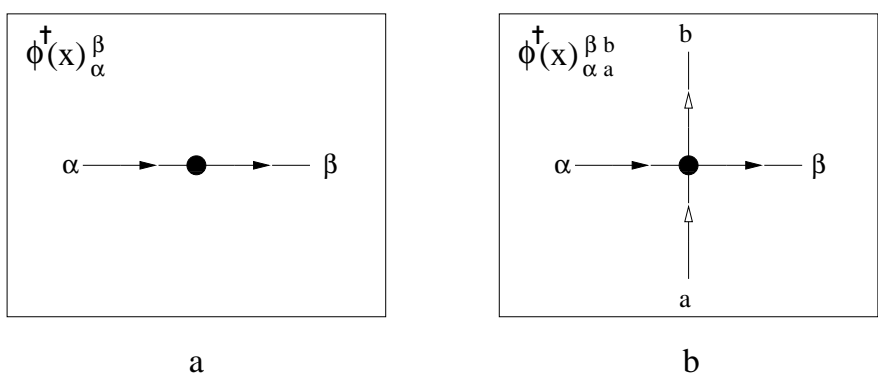

Figure 6: (a) String-bit (b) Membrane-bit

$\phi(x)_{\alpha a}^{\beta b}$, and the color group is enlarged to $U\left(N_{c}\right) \times U\left(N_{c}\right)$. Greek indices will be used for the first $U\left(N_{c}\right)$ factor, and Latin indices for the second. The bit-fields transform as the adjoint of each $U\left(N_{c}\right)$. The canonical commutator is given by

$$
\left[\phi(x)_{\alpha a}^{\beta b}, \phi^{\dagger}(y)_{\gamma c}^{\delta d}\right]=\delta_{\alpha}^{\delta} \delta_{\gamma}^{\beta} \delta_{a}^{d} \delta_{c}^{b} \delta(x-y) .
$$

The two $U\left(N_{c}\right)$ factors are distinct, and therefore so are the two sets of indices $(\alpha, \beta),(a, b)$. Consequently indices belonging to different $U\left(N_{c}\right)$ factors cannot be contracted. The generalization of the free Hamiltonian is straightforward :

$$
H_{0}=\frac{1}{2 m} \int d x \nabla \phi^{\dagger}(x)_{\alpha a}^{\beta b} \cdot \nabla \phi(x)_{\beta b}^{\alpha a} .
$$

Unlike string-bits, which could only form closed chains, membrane-bits can potentially form a rich variety of singlet structures, with the continuum properties of membranes, strings, or their combinations. The building blocks are tiles composed of a finite number of membrane-bits. The two possible pieces of string are shown in Fig.(7a), and possible pieces of membrane are shown in Fig.(7b). Fig.(7c) depicts tiles that can be sections of mixed structures. Closed membranes are

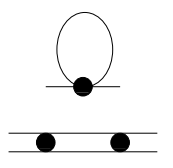

a

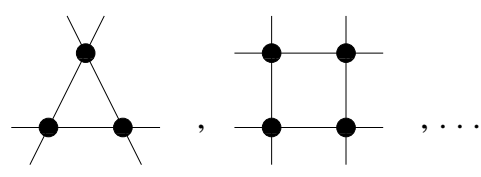

b

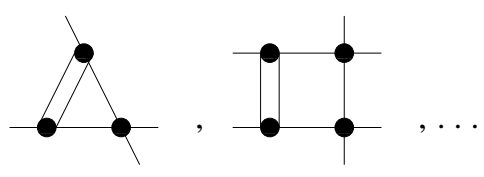

Figure 7: (a) String segments (b) Membrane tiles (c) Pieces of mixed structures

formed by fitting together the tiles in Fig.(7b) into compact (but not necessarily regular) lattices. 
This will in general require using more than one kind of tile. For examples of compact lattices and their continuum limits see Fig.(8). The figures represent ways of tracing over a product of tensor creation operators, and not the precise shape of the physical structure, since the bonds must all have equal length. The topology is however the same. Closed strings (chains) are formed by

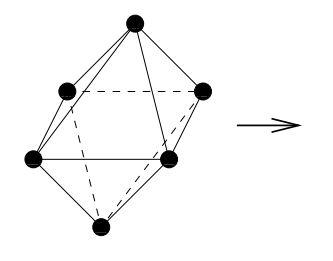

a

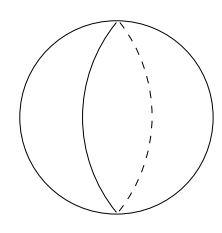

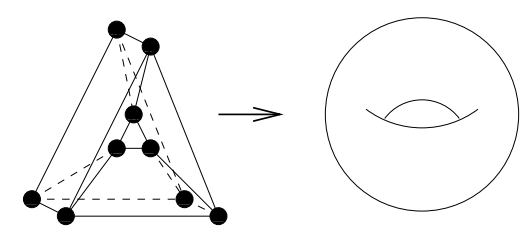

b

Figure 8: (a) Spherical membrane (b) Toroidal membrane

attaching either of the string segments of Fig.(7a) to each other (Fig.(9a)). Other stringy objects can be formed by combining the two kinds of segment (Fig.(9b,c)). Note that the continuum limit of Fig.(9b) looks like an open string whose ends are attached to a closed string, and the continuum limit of Fig.(9c) looks like an open string whose ends are attached to two different closed strings. Such configurations are familiar in the D-brane approach to 1-branes, which play the role of the closed string 16]. A generic membrane-bit model would include these configurations, and would therefore encompass the D-brane idea.
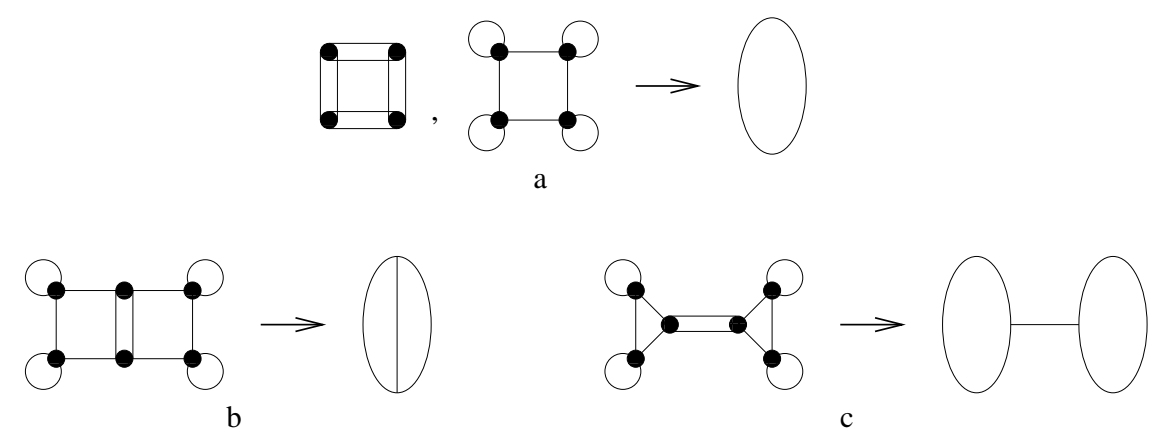

Figure 9: Stringy objects

A priori all of these structures are just mathematical (singlet) entities. Given a particular interaction term in the membrane-bit Hamiltonian, some of them will be actual physical structures. For these, the inter-bit connections correspond to physical bonds. The interaction should be general enough to allow the formation of both stringy and membrane-like discrete structures, but not necessarily all possible discrete structures. As in the bosonic string-bit model, we will consider only two-body interactions. Unlike the string-bit model however, there are many ways to form a connected singlet two-body interaction term. We do not intend to present an exhaustive analysis of two-body membrane-bit interactions. Instead we will give a taste of the richness of membrane-bit models by considering only the following three interactions:

$$
\begin{aligned}
H_{1} & =\frac{1}{N_{c}^{2}} \int d x d y V_{1}(x-y) \phi^{\dagger}(x)_{\alpha a}^{\beta b} \phi^{\dagger}(y)_{\beta b}^{\gamma c} \phi(y)_{\gamma c}^{\delta d} \phi(x)_{\delta d}^{\alpha a} \\
H_{2} & =\frac{1}{N_{c}} \int d x d y V_{2}(x-y) \phi^{\dagger}(x)_{\alpha a}^{\beta b} \phi^{\dagger}(y)_{\beta c}^{\delta d} \phi(y)_{\delta d}^{\epsilon c} \phi(x)_{\epsilon b}^{\alpha a}
\end{aligned}
$$




$$
H_{3}=\frac{1}{N_{c}} \int d x d y V_{3}(x-y) \phi^{\dagger}(x)_{\alpha a}^{\beta b} \phi^{\dagger}(y)_{\gamma b}^{\delta d} \phi(y)_{\delta d}^{\gamma c} \phi(x)_{\beta c}^{\alpha a}
$$

These interactions are represented graphically in Fig.(10). Note that the unfilled bits represent annihilation operators (removal of bits), whereas the filled bits represent creation operators. As in

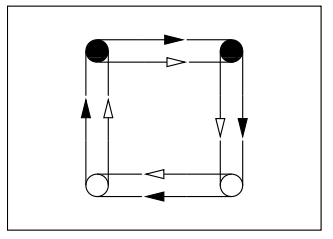

$H_{1}$

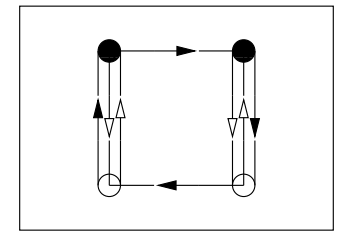

$\mathrm{H}_{2}$

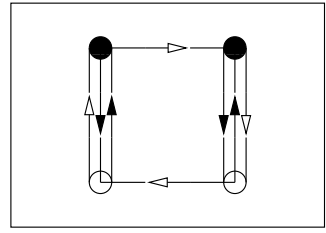

$\mathrm{H}_{3}$

Figure 10: Examples of two-body membrane-bit interactions

the string-bit models these interaction terms should act invariantly on singlet structures to lowest order in $1 / N_{c}$, and give rise to a nearest-neighbor interaction pattern. Since the interactions we are considering act on two bits at a time, it is sufficient to determine their action on connected 2-bit states, which can be embedded in larger singlet structures (chains or lattices). Of course just as there are many possible interaction terms, there are many possible 2-bit states. The possible 2-bit states are shown in Fig.(11), and are respectively given by

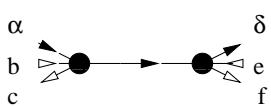

(a)

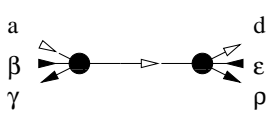

(b)

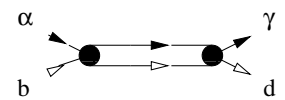

(c)

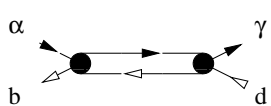

(d)

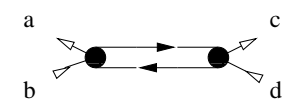

(e)

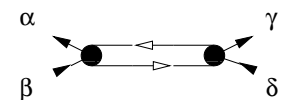

(f)

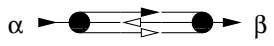

(g)

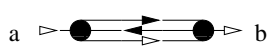

(h)

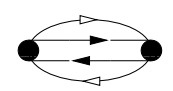

(i)

Figure 11: Connected 2-bit states
(a) $\phi^{\dagger}(x)_{\alpha b}^{\lambda c} \phi^{\dagger}(y)_{\lambda e}^{\delta f}|0\rangle$
(d) $\phi^{\dagger}(x)_{\alpha l}^{\lambda b} \phi^{\dagger}(y)_{\lambda d}^{\gamma l}|0\rangle$
(g) $\phi^{\dagger}(x)_{\alpha t}^{\lambda l} \phi^{\dagger}(y)_{\lambda l}^{\beta t}|0\rangle$
(b) $\phi^{\dagger}(x)_{\beta a}^{\gamma l} \phi^{\dagger}(y)_{\epsilon l}^{\rho d}|0\rangle$
(e) $\phi^{\dagger}(x)_{\lambda b}^{\theta a} \phi^{\dagger}(y)_{\theta d}^{\lambda c}|0\rangle$
(h) $\phi^{\dagger}(x)_{\lambda a}^{\theta t} \phi^{\dagger}(y)_{\theta t}^{\lambda b}|0\rangle$
(c) $\phi^{\dagger}(x)_{\alpha b}^{\lambda l} \phi^{\dagger}(y)_{\lambda l}^{\gamma d}|0\rangle$
(f) $\phi^{\dagger}(x)_{\beta l}^{\alpha t} \phi^{\dagger}(y)_{\delta t}^{\gamma l}|0\rangle$
(i) $\phi^{\dagger}(x)_{\alpha a}^{\beta b} \phi^{\dagger}(y)_{\beta b}^{\alpha a}|0\rangle$

Exchanging $x$ and $y$ yields new states, except for (e),(f) and (i), and we will denote them by the corresponding letter with a prime. We now act with each of the Hamiltonians in (6.3) on each of these states, keeping track only of the general structure, not of the precise indices. This will yield two terms for each state, corresponding to the two ways of contracting the two annihilation operators of the interactions with the two creation operators of the states. Every bond overlap will yield a factor of $N_{c}$. The results are summarized in the following table : 


\begin{tabular}{|c||c|c|c|}
\hline & $H_{1}$ & $H_{2}$ & $H_{3}$ \\
\hline \hline (a) & $N_{c}^{-1}(\mathrm{c})+N_{c}^{-2}\left(\mathrm{~h}^{\prime}\right)$ & $(\mathrm{a})+N_{c}^{-1}(\mathrm{e})$ & $N_{c}^{-1}[(\mathrm{c})+(\mathrm{d})]$ \\
\hline (b) & $N_{c}^{-1}(\mathrm{c})+N_{c}^{-2}\left(\mathrm{~g}^{\prime}\right)$ & $N_{c}^{-1}\left[(\mathrm{c})+\left(\mathrm{d}^{\prime}\right)\right]$ & $(\mathrm{b})+N_{c}^{-1}(\mathrm{f})$ \\
\hline (c) & $(\mathrm{c})+N_{c}^{-2}(\mathrm{i})$ & $(\mathrm{c})+N_{c}^{-1}(\mathrm{~h})$ & $(\mathrm{c})+N_{c}^{-1}\left(\mathrm{~g}^{\prime}\right)$ \\
\hline (d) & $N_{c}^{-1}\left[(\mathrm{~g})+\left(\mathrm{h}^{\prime}\right)\right]$ & $(\mathrm{d})+N_{c}^{-1}\left(\mathrm{~h}^{\prime}\right)$ & $N_{c}^{-1}(\mathrm{~g})+(\mathrm{d})$ \\
\hline (e) & $N_{c}^{-1}\left[(\mathrm{~h})+\left(\mathrm{h}^{\prime}\right)\right]$ & $2(\mathrm{e})$ & $N_{c}^{-1}\left[(\mathrm{~h})+\left(\mathrm{h}^{\prime}\right)\right]$ \\
\hline (f) & $N_{c}^{-1}\left[(\mathrm{~g})+\left(\mathrm{g}^{\prime}\right)\right]$ & $N_{c}^{-1}\left[(\mathrm{~g})+\left(\mathrm{g}^{\prime}\right)\right]$ & $2(\mathrm{f})$ \\
\hline (g) & $(\mathrm{g})+N_{c}^{-1}(\mathrm{i})$ & $(\mathrm{g})+N_{c}^{-1}(\mathrm{i})$ & $2(\mathrm{~g})$ \\
\hline (h) & $(\mathrm{h})+N_{c}^{-1}(\mathrm{i})$ & $2(\mathrm{~h})$ & $(\mathrm{h})+N_{c}^{-1}(\mathrm{i})$ \\
\hline (i) & $2(\mathrm{i})$ & $2(\mathrm{i})$ & $2(\mathrm{i})$ \\
\hline
\end{tabular}

In the limit $N_{c} \rightarrow \infty$, the states left invariant by $H_{1}, H_{2}$ and $H_{3}$ are respectively $\{(\mathrm{c}),(\mathrm{g}),(\mathrm{h}),(\mathrm{i})\}$, $\{(\mathrm{a}),(\mathrm{c}),(\mathrm{d}),(\mathrm{e}),(\mathrm{g}),(\mathrm{h}),(\mathrm{i})\}$ and $\{(\mathrm{b}),(\mathrm{c}),(\mathrm{f}),(\mathrm{g}),(\mathrm{h}),(\mathrm{i})\}$. When the interaction $H_{i}(i=1,2,3)$ acts on a large singlet structure in the limit $N_{c} \rightarrow \infty$, one gets a nearest-neighbor interaction $V_{i}$ for every membrane-bit pair belonging to the corresponding set. With more than one of the interaction terms present, some of the membrane-bit pairs will acquire a sum of interactions. For example, if the membrane-bit model had the following interaction

$$
H=\lambda_{1} H_{1}+\lambda_{2} H_{2}+\lambda_{3} H_{3}
$$

the membrane-bit pair (c) would interact through $\lambda_{1} V_{1}+\lambda_{2} V_{2}+\lambda_{3} V_{3}$, whereas the pair (a) would only interact through $\lambda_{2} V_{2}$, and the pair (b) would only interact through $\lambda_{3} V_{3}$.

Pure membranic structures contain only single bonds, and therefore contain only the pairs (a) and (b). Consequently if $\lambda_{2}=\lambda_{3}=0$, the model would not support pure membrane formation, since (a) and (b) are not in the invariant set of $H_{1}$. Closed chains on the other hand can be singlebonded or double-bonded (Fig.(9a)). For these values of the parameters only the double-bonded variety would be supported. At generic values of the parameters, both strings and membranes, as well as mixed structures, are possible. A reasonable (but not necessary) assumption is that the bonds all carry the same interaction, $V_{1}(x) / 2=V_{2}(x)=V_{3}(x)=V(x)$. The parameter space spanned by $\lambda_{1}, \lambda_{2}, \lambda_{3}$ then serves as a kind of "moduli space". At a generic point all structures can exist, but there are regions where only some of the structures will form. Of course there are many more possible membrane-bit interaction terms, and therefore many more parameters in this "moduli space".

The generalization to higher dimensional $p$-branes consists of adding more pairs of legs to the bits. A $p$-brane bit will have $2 p$ legs. Such bits would be able to form not only $p$-branes, but by utilizing self-contractions, double-contractions, triple-contractions, etc., would also be able to form all the lower branes, as well as various mixed structures.

The 0-brane is somewhat puzzling. The only candidate seems to be a single bit with all its legs self-contracted. But as a single bit, this object should disappear in the continuum limit.

\section{Discussion}

All of the evidence presented here, together with further investigations of string dualities, suggest that the true formulation of string theory, if it exists, is radically different from the world-sheet formulation. We have considered the possibility that string theory is in fact a low energy effective theory of a more fundamental theory of constituents. String-bit models are an appealing idea because they provide a microscopic origin for both the curious high temperature behavior and the 
high longitudinal boost behavior of string theory. They predict a dissociation phase transition at $T_{c} \sim T_{0} / m$, and can result in different high $p^{+}$behaviors depending on the details of the microscopic bit interactions. Agreement with the black hole entropy formula seems to imply that there is a maximum density of bits, but it is not yet clear which string-bit model will achieve this.

The main drawback of string-bit models is that there are so many of them. There is a large universality class of models that leads to the same free string limit. Comparing with known string interactions may fix some of the ambiguity. In fact it was shown in the supersymmetric case that a rather specific form of bit interaction was required in order to prevent long range interactions between separated chains. However, it is not clear yet whether there is a unique superstring-bit model.

It is also not clear how the role of the dilaton as the string coupling constant is realized in string-bit models, given that $g \sim 1 / N_{c}$.

Finally, we have seen that membranes and other extended objects could in principle be formed from constituents as well. Given bits that have $2 p$ legs, and sufficient two-body interactions, one can form extended structures of dimension $\leq p$. The various two-body interactions span a "moduli space" containing degenerate regions, where some of the structures are not supported. This bears close resemblance to the idea of " $p$-brane democracy" that asserts that in the fundamental formulation all $p$-branes are equally fundamental. It is only when we look at a special region of moduli space that the string appears to be more fundamental.

\section{Acknowledgments}

This work was supported in part by the Department of Energy under grant DE-FG05-86ER-40272 and by the Institute for Fundamental Theory. I wish to thank Charles Thorn and Larus Thorlacius for useful discussions.

\section{References}

[1] J. J. Atick and E. Witten, Nucl. Phys. B310 (1988) 291.

[2] L. Susskind, "The World as a Hologram", J. Math. Phys. 36 (1995) 6377, hep-th/9409089; "Strings, Black Holes and Lorentz Contractions", Phys. Rev. D49 (1994) 6606, hepth/9308139; "String Theory and the Principles of Black Hole Complementarity", Phys. Rev. Lett. 71 (1993) 2367, hep-th/9307168.

[3] C.M. Hull and P.K. Townsend, "Unity of Superstring Dualities", QMW-94-30, R/94/33, hepth/9410167.

[4] E. Witten, "String Theory Dynamics in Various Dimensions", Nucl. Phys. B443 (1995) 85, hep-th/9503124.

[5] J.H. Schwarz, "The Power of M-theory", hep-th/9510086.

[6] P. Hořava and E. Witten, "Heterotic and Type I String Dynamics from Eleven Dimensions", hep-th/9510209.

[7] P.K. Townsend, "P-Brane Democracy", hep-th/9507048. 
[8] C. B. Thorn, "Reformulating String Theory with the 1/N Expansion," in Sakharov Memorial Lectures in Physics, Ed. L. V. Keldysh and V. Ya. Fainberg, Nova Science Publishers Inc., Commack, New York, 1992; hep-th/9405069.

[9] C. B. Thorn, "Calculating the Rest Tension for a Polymer of String Bits", Phys. Rev. D51 (1995) 647, hep-th/9407169.

[10] O. Bergman and C. B. Thorn, "Superstring-Bit Models for Superstring", Phys. Rev. D52 (1995) 5980, hep-th/9506125.

[11] O.Bergman and C. B. Thorn, "Universality and Clustering in 1+1 Dimensional Superstring-Bit Models", Phys. Rev. Lett. 76 (1996) 2214, hep-th/9512107.

[12] C. B. Thorn, Phys. Lett. 99B (1981) 458.

[13] D.J. Gross and P.F. Mende, Nuc. Phys. B303 (1988) 407.

[14] G. 't Hooft, "Dimensional Reduction in Quantum Gravity," Utrecht preprint THU-93/26, gr-qc/9310026.

[15] M. Duff, R. Khuri, and J. Lu, Phys. Rep. 259 (1995) 213, hep-th/9412184, and references therein.

[16] J. Polchinski, "Dirichlet-Branes and Ramond-Ramond Charges", NSF-ITP-95-122, hepth/9510017.

[17] P.K. Townsend, "The Eleven Dimensional Supermembrane Theory Revisited", R/95/2, hepth/9501068.

[18] G.F. Chew, "The Analytic S matrix: a basis for nuclear democracy", New York, W.A. Benjamin, 1966.

[19] M. Gell-Mann and Y. Ne'eman, "The Eightfold Way", New York, W.A. Benjamin, 1964.

[20] O. Bergman and C. B. Thorn, "Super-Galilei Invariant Field Theories in 2+1 Dimensions", Phys. Rev. D52 (1995) 5997, hep-th/9507007.

[21] R. Giles, L. McLerran, and C. B. Thorn, Phys. Rev. D17 (1978) 2058.

[22] E. Witten, Nuc. Phys. B188 (1981) 513.

[23] M. B. Green, J. H. Schwarz, and L. Brink, Nucl. Phys. B219 (1983) 437. 